\title{
Biopsy and personalized medicine
}

David C. Whitcomb and Adrian V. Lee

In their correspondence, Amalou and Wood rightly highlight the transition of advanced biopsy and imaging techniques from diagnostic to personalized prognostic tools. ${ }^{1}$ My Perspectives article ${ }^{2}$ used as its illustrative model the non-neoplastic disease of pancreatitis, in which determining susceptibility attributable to germ line variations in the DNA sequence is more informative than examining pancreatic tissue, whether from biopsy or on imaging. However, as Amalou and Wood point out, in the area of oncology, advanced imaging and biopsy techniques for next-generation 'omics' analyses will have a major role in the ultimate delivery of personalized medicine. For example, studies published in the past 2 years that examined the heterogeneity and evolution of somatic DNA changes in pancreatic ${ }^{3}$ and other cancers ${ }^{4}$ have revealed dynamic remodelling of the cancer genome. In this instance, further development and implementation of such imaging and biopsy techniques will support the development of predictive modelling that might help us to identify patients with pancreatic cancer early and to improve their treatment. I also agree that a focus on the tumour tissue itself will be essential for drug discovery and personalization in cancer care, although a parallel benefit does not exist in the chronic inflammatory conditions that served as the focus of my article.

Division of Gastroenterology, Hepatology and Nutrition, University of Pittsburgh \& UPMC, Room 401.4, 3708 Fifth Avenue, Pittsburgh, PA 15213, USA (D. C. Whitcomb). Women's Cancer Research Center, University of
Pittsburgh Cancer Institute, Magee Women's Research Institute, 204 Craft Avenue, Room A412, Pittsburgh, PA 15213, USA (A. V. Lee). Correspondence to: D. C. Whitcomb whitcomb@pitt.edu

doi:10.1038/nrgastro.2012.100-c2

Competing interests

The authors declare no competing interests.

1. Amalou, H. \& Wood, B. J. Biopsy and personalized medicine. Nat. Rev. Gastroenterol. Hepatol. http://dx.doi.org/10.1038/ nrgastro.2012.100-c1.

2. Whitcomb, D. C. What is personalized medicine and what should it replace? Nat. Rev. Gastroenterol. Hepatol. 9, 418-424 (2012).

3. Campbell, P. J. et al. The patterns and dynamics of genomic instability in metastatic pancreatic cancer. Nature 467, 1109-1113 (2010).

4. Gerlinger, M. et al. Intratumor heterogeneity and branched evolution revealed by multiregion sequencing. N. Engl. J. Med. 366, 883-892 (2012). 